\title{
Transposition
}

Musique et Sciences Sociales

1 | 2011

Polyphonie et société

\section{L'enseignement du contrepoint et de la fugue au Conservatoire de Paris (1858-1905)}

Teaching Counterpoint and Fugue at the Conservatoire de Paris (1858-1905)

\section{Anthony Bergerault}

\section{(2) OpenEdition}

\section{Journals}

Édition électronique

URL : http://journals.openedition.org/transposition/418

DOI : 10.4000/transposition.418

ISSN : 2110-6134

Éditeur

CRAL - Centre de recherche sur les arts et le langage

Référence électronique

Anthony Bergerault, «L'enseignement du contrepoint et de la fugue au Conservatoire de Paris (1858-1905) », Transposition [En ligne], 1 | 2011, mis en ligne le 01 février 2011, consulté le 01 mai 2019. URL : http://journals.openedition.org/transposition/418; DOI : 10.4000/transposition.418

Ce document a été généré automatiquement le 1 mai 2019.

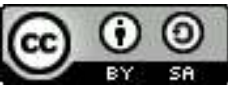

La revue Transposition est mise à disposition selon les termes de la Licence Creative Commons Attribution - Partage dans les Mêmes Conditions 4.0 International. 


\title{
L'enseignement du contrepoint et de la fugue au Conservatoire de Paris (1858-1905)
}

Teaching Counterpoint and Fugue at the Conservatoire de Paris (1858-1905)

\author{
Anthony Bergerault
}

1 Malgré son importance dans la vie musicale française et les ouvrages parus à l'occasion de son bicentenaire ${ }^{1}$, le Conservatoire de Paris demeure une institution peu étudiée. Nous allons tenter ici de restituer la manière dont les études de contrepoint et de fugue y ont été organisées, notamment en ce qui concerne l'aspect pédagogique de ces disciplines, grâce à l'exploitation et au croisement des sources contenues dans les archives du Conservatoire, largement méconnues.

Dans la seconde moitié du XIX ${ }^{e}$ siècle, ces enseignements sont indissociables de celui de la composition. Créées en 1818, les deux classes de contrepoint et fugue - dans lesquelles ces deux disciplines étaient enseignées conjointement - sont réunies en 1840 aux deux classes de composition. A partir de cette date, il y a eu au sein du Conservatoire quatre classes ${ }^{2}$ dites de composition uniquement, réunissant en réalité sous une dénomination unique les trois disciplines. En 1878 y sera adjoint l'enseignement de l'instrumentation. Durant la période qui nous intéresse, le contrepoint et la fugue se trouvent donc enseignés par un pédagogue aux attributions multiples et sont un point incontournable de la formation de tous les compositeurs qui ont étudié au Conservatoire.

Cette proximité qui témoigne de l'importance accordée à cette époque à la compétence technique dans la création musicale n'est pas sans inconvénients pour le chercheur d'aujourd'hui. La forte valorisation de la dimension compositionnelle, qui constitue évidemment le couronnement de ces études, jette une ombre certaine sur les autres aspects de la formation. Le concours pour les prix de contrepoint et fugue, organisé par le Conservatoire, pâtit grandement de la notoriété du Grand Prix de Rome, concours de composition qui dépend de l'Académie des Beaux-Arts. Ce dernier monopolise l'attention de la presse, focalise aussi les témoignages ultérieurs des musiciens issus de ce système 
sur leurs années de formation. Le recrutement des enseignants, au nombre desquels comptent Massenet, Delibes, Fauré, Widor ${ }^{3}$, se fait en premier lieu en fonction de leur carrière en tant que compositeurs. Dans ce contexte, les traces concrètes de l'activité au quotidien des classes dans le domaine qui nous préoccupe se font rares.

Néanmoins, grâce aux devoirs d'élèves produits dans le cadre du concours pour le prix de contrepoint et fugue, il est possible d'accéder à un état du travail effectué dans ces classes. Certes aboutis mais moins contrôlés et moins idéaux que dans les traités, ces exercices peuvent également être confrontés au jugement porté par les jurys à l'époque, ce qui devrait permettre d'éviter les dangers d'un regard anachronique sur une discipline ayant évolué depuis cette époque. La conservation de ces devoirs dans les archives n'a commencé qu'en 1858, date à partir de laquelle le fonds se révèle quasiment exhaustif. Aussi avons-nous choisi de commencer notre étude à partir de cette date.

5 L'année 1905 voit un certain nombre de changements dans l'organisation institutionnelle des enseignements qui nous préoccupent. Lors de son accession à la direction de l'établissement, Gabriel Fauré réorganise les classes de composition en créant une classe de contrepoint confiée à Georges Caussade et une classe de fugue confiée à André Gedalge. Les professeurs de composition n'enseigneront plus ces disciplines. D'autre part, le prix de contrepoint et fugue est supprimé au profit d'un prix de contrepoint et d'un prix de fugue distincts. L'évolution des structures pédagogiques que cela suppose est suffisamment significative pour justifier d'en faire l'autre limite chronologique de notre travail.

6 Nous allons tenter de restituer ici la démarche pédagogique qui a présidé à l'enseignement de ces disciplines et ses applications concrètes en situation de concours, après avoir retracé dans un premier temps le contexte institutionnel dans lequel s'est effectué le parcours de étudiants.

\section{L'organisation des études}

\section{Dispositions réglementaires}

7 L'accès à la classe de composition se fait dans le cadre d'un parcours normatif très strict, encadré par les règlements de $1850^{4}$ et $1878^{5}$, qui prévoient un certain nombre de compétences à acquérir préalablement ou parallèlement. L'enseignement dispensé par le Conservatoire se structure dans le cursus qui nous intéresse en trois grandes étapes: solfège, harmonie puis enfin composition. La chronologie des études a également été pensée de manière attentive, puisqu'il est interdit de cumuler deux de ces enseignements. Les dispositions réglementaires sont en particulier très strictes sur la nécessité d'avoir achevé les études d'harmonie avant de commencer celles de composition, donc également de contrepoint et de fugue. Cette structuration de l'enseignement se retrouve dans les traités : l'harmonie fait l'objet d'ouvrages autonomes alors que le contrepoint et la fugue sont fréquemment associés en une publication unique.

8 En revanche, lorsque l'étudiant a fait la preuve de ses connaissances, essentiellement dans le domaine de l'harmonie - sans qu'il lui soit nécessaire pour autant d'avoir obtenu un prix au concours de fin d'année - il peut obtenir son passage en classe de composition par simple autorisation du directeur, sans qu'il faille réunir un jury pour avaliser cette admission, contrairement à la majorité des classes de l'établissement, notamment les 
classes instrumentales. L'exigence portée sur la formation préalable des aspirants joue un rôle modérateur et garantit leur niveau technique, ce qui permet d'alléger les formalités pour accéder à l'enseignement de la composition.

9 Par ailleurs, le cursus s'enrichit en 1878 d'un nouvel enseignement parallèle, celui de l'histoire de la musique. Ce cours, créé quelques années auparavant, est rendu obligatoire aux élèves d'harmonie et de composition. Ce sont les seules classes soumises à cet impératif, ce qui dénote le souci de garantir une certaine érudition aux futurs créateurs. Il existe également des classes d'étude du clavier réservées aux élèves de chant, d'harmonie et de composition. Pour les étudiants non claviéristes, l'acquisition d'un minimum de technique permettant de lire de la musique au piano semble nettement encouragée.

On voit donc que l'enseignement du contrepoint et de la fugue au Conservatoire a été envisagé au sein d'une formation riche et complète, organisée pour mener vers le métier de compositeur. Les disciplines qui nous intéressent sont dans ce cursus les plus étroitement associées à l'étude de la composition proprement dite et constituent l'un des sommets de cette formation.

\section{Parcours instrumental des lauréats ${ }^{6}$}

11 Si nous nous intéressons aux récompenses instrumentales obtenues dans l'établissement par les lauréats de contrepoint et fugue, nous constatons une nette prédominance d'étudiants pianistes et organistes, ce qui relativise l'importance de la classe optionnelle d'étude du clavier, laquelle ne concerne en réalité qu'une petite minorité des effectifs. D'après le Dictionnaire des lauréats établi par Pierre Constant ${ }^{7}$, sur la période allant de 1858 à 1900, 71 des 137 lauréats de contrepoint et fugue apparaissent également dans les palmarès instrumentaux. Nous n'indiquerons donc ici que des tendances générales, puisque ces informations ne recouvrent que la moitié environ des lauréats.

12 Sur les 71 étudiants dont nous connaissons le parcours instrumental, on dénombre 59 claviéristes, 8 violonistes, 3 flûtistes et 1 harpiste. Parmi les $80 \%$ d'étudiants pratiquant un instrument à clavier, la répartition entre l'étude du piano et celle de l'orgue est plutôt équilibrée: respectivement 38 et 35 lauréats, dont 14 récompensés dans les deux disciplines. Cette relative égalité marque la proximité privilégiée entre les études d'orgue et de composition, puisque les pianistes sont au sein du Conservatoire considérablement plus nombreux que les organistes, sans que le cursus de composition se ressente de ce déséquilibre.

13 La très faible diversité des instruments pratiqués par les élèves de contrepoint et fugue est révélatrice d'une hiérarchie dans la pensée musicale. La primauté indéniable des instruments polyphoniques s'accompagne d'un modèle compositionnel où la prédominance de la mélodie dans le discours est révélée par la présence des dessus principaux de l'orchestre, le violon essentiellement et la flûte dans une moindre mesure, alors qu'aucun des autres instruments n'est représenté. L'étude du contrepoint et de la fugue vise justement à tempérer, à contrôler cette dimension mélodique pour enrichir la pensée musicale par la maîtrise de techniques de composition à voix d'égale importance. 


\section{Les examens et concours}

\section{Examens semestriels}

14 A l'instar des autres classes, les étudiants en composition sont évalués deux fois par an par un jury dont les membres sont nommés par décret ministériel. Les élèves présentent des exercices de contrepoint, des fugues et des compositions libres, reflétant la diversité des enseignements de la classe ${ }^{8}$. Le premier de ces examens a lieu en janvier, le second en juin.

A cette occasion, les professeurs rendent un rapport sur l'activité de chaque élève au sein de la classe. Ces documents constituent aujourd'hui un témoignage intéressant du travail effectué en cours, quoique le contenu et la précision des commentaires soient très variables d'un professeur à l'autre. Voici à titre d'exemple le rapport de Fauré sur son élève Ravel en janvier $1899^{9}$ :

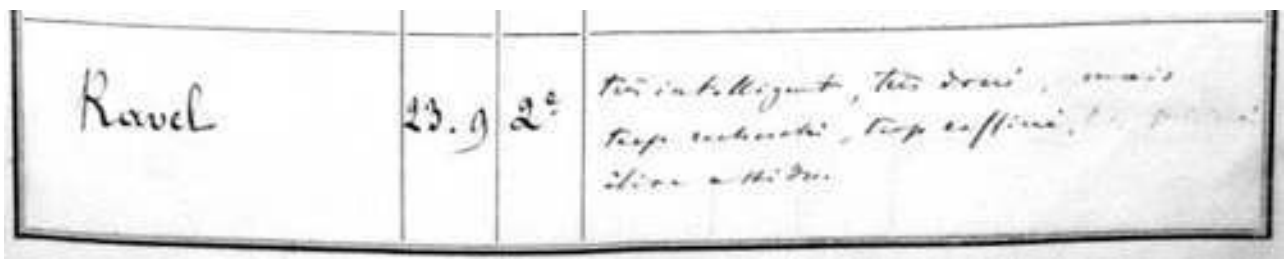

\section{Concours annuel des prix}

La principale évaluation reste le concours pour les prix de contrepoint et fugue, qui se déroule annuellement durant la première quinzaine du mois de juillet. Les candidats autorisés à concourir sont désignés à l'issue de l'examen semestriel de juin par le jury. En sont donc exclus d'une part les élèves n'étant pas jugés assez avancés dans leurs études et d'autre part ceux se consacrant désormais totalement à la composition. L'objet du concours est la réalisation d'une fugue vocale à quatre parties dans le style d'école. Ce travail s'effectue en loge, au Conservatoire. La durée de l'épreuve est de dix-huit heures le dimanche de six heures du matin à minuit - et l'usage du piano est interdit. Ce sont du reste des conditions d'examen assez similaires à ce qui se pratique encore aujourd'hui pour les classes d'écriture au Conservatoire de Paris. Cette organisation immuable persiste donc bien au-delà de la période que nous traitons et de nombreux éléments subsistent encore de nos jours.

Comme pour la plupart des autres classes, le Conservatoire distribue à l'issue du concours des récompenses dénommées prix et accessits. En 1858, ces récompenses au nombre de cinq se répartissent par ordre décroissant en premier prix, second prix, premier accessit, second accessit et troisième accessit. Seul le premier prix pouvait être décerné à deux lauréats la même année. Les membres du jury étaient libres également de ne pas décerner certaines de ces récompenses s'ils estimaient qu'aucun concurrent ne la méritait. Le troisième accessit a été supprimé en 1873 mais chaque récompense pouvait dorénavant être décernée deux fois. Pour quatre récompenses, on verra fréquemment six ou sept lauréats par an à la fin du siècle, et l'attribution possible à trois concurrents de chaque récompense après 1900 fera s'étendre le palmarès jusqu'à dix lauréats en 1905 alors que les effectifs n'augmentent pas dans la même proportion. La tendance va donc vers une 
distribution de plus en plus généreuse et une reconnaissance croissante du parcours des musiciens dans ces classes.

Le jury chargé de décerner les récompenses dont nous venons de parler se réunit dans la semaine qui suit la mise en loge, généralement le lundi ou le mardi ${ }^{10}$. Le délai entre la réalisation de l'épreuve et l'établissement du palmarès est donc aussi réduit que possible. Le jugement se fait de manière anonyme: chacune des copies porte la signature du candidat (masquée par une bande de papier collé la recouvrant) et un numéro qui sert à l'identifier pour la procédure de vote. Le nombre de membres composant le jury est habituellement de neuf, dont le directeur du Conservatoire qui le préside. Les professeurs ne peuvent évidemment faire partie du jury de leur propre classe. Après avoir procédé à l'examen des copies à la table, les jurés peuvent exécuter celles de leur choix au piano. Il est ensuite procédé au scrutin à bulletin secret.

Nous ne détaillerons pas ici le système de vote, assez élaboré, mais il n'est pas douteux. Les procès-verbaux dressés par le secrétaire, source d'informations incontournable sur le déroulement de la séance, ne reflètent pas dans leur précision méticuleuse l'atmosphère de la salle mais plutôt un fonctionnement administratif parfaitement rodé. Les pratiques de sociabilité de musiciens pratiquant le même métier, ayant de surcrôit la même spécialisation, devaient également constituer par le biais d'échanges verbaux une composante majeure du déroulement du jugement, ne serait-ce que par le choix en commun des fugues à entendre au piano qui forment ensuite généralement la tête du palmarès.

\section{Les traces de l'enseignement}

Il est difficile de trouver des informations sur le travail pédagogique dans le cadre de la classe. La vocation d'un exercice de contrepoint ou de fugue n'est pas, contrairement à certains devoirs de composition, d'être conservé pour des exécutions du vivant de l'auteur ou pour un éventuel passage à la postérité. De plus, l'ombre jetée sur le travail de la fugue par la prédominance de la composition rend les témoignages sur cet aspect de l'enseignement particulièrement rares, les auteurs s'étendant volontiers dans leur correspondance ou leurs mémoires sur la présentation en classe des travaux de composition, mais délaisseront les autres aspects de l'enseignement.

21 Toutefois, dans la préface du traité de fugue de Gedalge ${ }^{11}$, l'auteur évoque « un contact journalier avec les élèves, alors que je suppléais mon maître Ernest Guiraud ou que M. Massenet me faisait l'honneur de me confier, dans sa classe, les études de contrepoint et de fugue ». Des classes préparatoires de contrepoint et de fugue, confiées à de jeunes professeurs tout récemment sortis des classes principales, ont existé entre 1819 et 1840, mais une telle pratique semble d'après les documents administratifs avoir disparu après cette date. Nous constatons donc que contrairement aux informations officielles un tel usage a pu se perpétuer librement dans certaines classes, au gré des occasions.

A défaut de s'appuyer sur des témoignages ou des devoirs réalisés en cours d'année pour reconstituer l'organisation du travail pédagogique de la fugue, deux types de documents constituent des sources de tout premier ordre pour contribuer à combler cette lacune : les traités publiés par des professeurs du Conservatoire et les devoirs réalisés en loge pour le concours annuel des prix, qui font l'objet d'un archivage presque systématique au cours de notre période d'étude. Nous tenterons d'éclairer ces documents à l'aide des 
renseignements que l'on peut tirer des notes prises par les membres du jury à l'occasion des examens semestriels.

\section{Les traités de contrepoint et de fugue ${ }^{12}$}

23 Aux origines du Conservatoire, le règlement de $1800^{13}$ établissait l'obligation pour les professeurs de l'institution de se consacrer à la rédaction d'ouvrages de référence pour l'enseignement. Plusieurs traités paraitront donc, dus à la plume d'enseignants (ou anciens enseignants) du Conservatoire. Ces publications répondent néanmoins tardivement à l'exigence initiale, puisqu'il faut attendre les années 1820 pour que les premiers ouvrages voient le jour.

Voici la liste des ouvrages concernant le contrepoint et la fugue parus jusqu'en 1905 dont les auteurs ont enseigné cette discipline au Conservatoire :

- Anton Reicha, Traité de haute composition musicale, Paris, Zetter, 1824.

- François-Joseph Fétis, Traité du contrepoint et de la fugue, Paris, Troupenas, 1825.

- Luigi Cherubini, Cours de contrepoint et de fugue, Paris, Schlesinger, 1835.

- François Bazin, Cours de contrepoint théorique et pratique, Paris, Lemoine, 1881.

- Théodore Dubois, Traité de contrepoint et de fugue, Paris, Heugel, 1901.

- André Gedalge, Traité de la fugue, Paris, Enoch, 1901.

\section{Le style de référence : idéal et contradictions}

Les fondements musicaux de l'enseignement reposent sur des répertoires assez divers. $\mathrm{Si}$ tous s'accordent à reconnaître en Jean-Sébastien Bach un modèle pour l'enseignement de la fugue, les références concernant l'étude du contrepoint sont un peu plus floues, désignées de surcroît par des termes imprécis: pour Cherubini ce sont les classiques, Dubois parlant plutôt d'école palestrinienne, mais les limites historiques et stylistiques des œuvres de référence ne sont pas clairement posées. Toutefois, les références au traité de Fux par Cherubini cernent bien cette esthétique musicale assez large qui recouvre la seconde moitié du $\mathrm{XVI}^{\mathrm{e}}$ siècle et le début du XVII ${ }^{\mathrm{e}}$ siècle, dans les œuvres religieuses chorales.

Cependant, dans le prolongement de l'apprentissage effectué en classe d'harmonie, le système musical utilisé dans les exercices de contrepoint du Conservatoire est entièrement tonal. Toute tournure étrangère aux lois de la tonalité est bannie du langage, même si elle était usitée par les compositeurs de référence. Palestrina en particulier se sert souvent de l'enchaînement contrapuntique de la tierce majeure vers la quinte, provoquant un mouvement harmonique de la dominante vers la sous-dominante. Ce procédé d'écriture sera proscrit par tous les traités que nous avons cités, pour des raisons liées à l'emploi du système tonal, au mépris de l'authenticité historique.

La démarche suivie consiste donc à tirer du panel d'œuvres de référence des principes généraux, lesquels, après abstraction de leur contexte originel pour les considérer comme absolus, sont réutilisés, ré-appliqués dans un système théorique différent. Dans l'enseignement de la fugue, ce paradoxe s'exprime à trois niveaux : il s'agit de produire une œuvre selon les canons formels de Bach en utilisant les ressources de la combinatoire contrapuntique du XVIe siècle, le tout dans un contexte harmonique tonal du XIX siècle. La contradiction qui s'établit alors entre les différents systèmes d'écriture musicale rend inopérante toute considération de style, la démarche pédagogique consistant plutôt en 
l'acquisition de procédés puisés à plusieurs sources. L'objet musical qui en résulte est synthétique, mais en revanche dépourvu de dimension historique.

Les phénomènes que nous venons de décrire provoquent une discordance par rapport aux répertoires authentiques qui seront cités, car ils ne peuvent correspondre entièrement à l'idéal recherché. Ils ouvrent la voie à toutes les critiques de la part des auteurs. Fétis assortit la citation d'un contrepoint de L'art de la fugue du commentaire suivant: "Ce passage [...] est écrit d'une manière fort incorrecte $»^{14}$. Chez Cherubini et Dubois, la reproduction d'extraits issus du répertoire ancien n'intervient que pour signaler des licences à ne surtout pas imiter. De plus, les œuvres d'où les passages incriminés ont été tirés ne sont même pas identifiées.

L'initiateur de cet éloignement du répertoire historique semble être Reicha. Dans son traité, le plus ancien de tous, il adopte le parti pris le plus radical et choisit délibérément d'exclure tout recours aux œuvres du passé. Les exemples utilisés dans son ouvrage sont tous de sa main, et comme il le dit lui-même dans sa préface, «faits tout exprès pour la matière traitée $»^{15}$. Cela a au moins le mérite de garantir l'adéquation entre les principes exposés et les illustrations proposées. A l'exception du traité de Fétis, paru très peu de temps après, les ouvrages ultérieurs s'inscriront dans la continuité de la démarche initiée par Reicha.

30 À la fin du siècle, le répertoire des exemples musicaux distincts des réalisations de l'auteur reprend pourtant sa place, mais il ne s'agit aucunement d'un retour vers les modèles anciens. Dans le traité de Dubois, ces exemples sont relégués à la fin de l'ouvrage. Il s'agit de devoirs d'élèves pour les concours du Conservatoire ayant remporté le premier prix. Le répertoire exogène est donc banni au profit d'un répertoire endogène, suscité par l'institution elle-même dans le cadre de son fonctionnement.

\section{La fixation d'un modèle pédagogique}

Ce revirement des références est absolument central pour comprendre l'orientation pédagogique. A l'origine il s'agissait, par un système inspiré des grandes œuvres du passé, d'atteindre une forme d'idéal dans la composition en allant extraire les procédés jugés les plus intéressants pour en tirer des principes d'écriture. L'institutionnalisation qui s'exerce par le biais du concours annuel conduit au bouleversement des valeurs : le temps passant, les valeurs de référence deviennent les devoirs primés, dignes d'être cités en modèle aux étudiants des générations futures car ils contiennent les qualités propres à satisfaire un jury. Les travaux de leurs aînés sont présentés aux étudiants comme un objectif à atteindre à l'issue de leur scolarité dans la perspective d'obtenir une bonne récompense. La pesanteur de l'institution déplace le centre d'intérêt du répertoire réel vers de la «fausse " musique de circonstance, produite pour le besoin de l'évaluation, soumise à des normes imposées. Les réalisations d'auteur dans les traités constituent l'étape intermédiaire de ce glissement. L'enseignement du contrepoint et de la fugue au $\mathrm{XIX}^{\mathrm{e}}$ siècle peut donc être lu comme la constitution progressive d'une entité autonome produisant le matériau nécessaire pour entretenir son propre fonctionnement. Le monde du contrepoint et de la fugue fonctionne alors en vase clos autour de valeurs de référence endogènes, cristallisées et immuables. 


\section{Etude des fugues de concours ${ }^{16}$}

Précisons d'ailleurs que la vocalité des fugues n'est que fictive. Les clés et ambitus vocaux doivent être respectés, mais les compositions n'ont aucune vocation à être chantées. L'absence de tout texte est significative à cet égard. Une fugue " vocale » n'est en aucune façon une fugue "pour la voix ». L'idée de registre vocal à l'intérieur de la tessiture autorisée est totalement évacuée de tous les traités, ce que l'objectif d'une exécution devrait normalement prendre en compte. Nous avons vu qu'à l'occasion du jugement, certains devoirs étaient lus au piano: cet instrument était leur seule occasion d'être interprétés. Nous verrons en abordant les sujets donnés au concours que l'idée même de vocalité de la ligne tend à disparaître en avançant dans la seconde moitié du XIX ${ }^{\mathrm{e}}$ siècle, pour ne plus recouvrir que la notion de "partie réelle », l'hypothétique exécution par des chanteurs étant compromise par certaines tournures, certains intervalles utilisés qui, bien que restant dans les tessitures usuelles, seraient bien inconfortables à chanter. Il s'agit bien là d'une musique abstraite et idéale, éloignée de toute préoccupation de réalisation sonore effective.

\section{Les sujets donnés}

Depuis le directorat de Cherubini, il était de tradition que le sujet donné au concours pour les prix de contrepoint et fugue soit composé par le directeur du Conservatoire. Une exception sera faite en 1896, année où le sujet sera commandé à Saint-Saëns, en raison du décès d'Ambroise Thomas en cours d'année. Contrairement à ce qui pouvait se pratiquer dans la première moitié du XIX ${ }^{e}$ siècle, le sujet donné change systématiquement d'une année sur l'autre, composé spécialement pour le concours. Il pourra toutefois servir pour les exercices des élèves des générations suivantes, l'habitude ayant été prise de recueillir les sujets de concours tant du Conservatoire que de l'Institut dans les traités ou des ouvrages spécifiques ${ }^{17}$.

\section{Les usages tacites}

Les fugues sont toujours écrites dans les clés usuelles du quatuor vocal, à savoir ut 1 , ut 3 , ut 4 , et fa 4 . Afin de gagner du temps - et parce que ces usages étaient à ce point ancrés dans les mœurs qu'ils en devenaient des automatismes -, une large partie des candidats omet de répéter les clés et les armures au-delà du premier système. L'usage conventionnel supplée naturellement à ce manque.

Nous avons vu que le jury lisait les copies à la table dans un premier temps, avant de sélectionner celles qu'il souhaitait entendre au piano. Afin de mieux faire valoir leur travail, et de rendre la structure formelle visuellement évidente, les candidats ont adopté l'habitude de signaler l'apparition de chaque élément thématique, en indiquant s'il s'agit du sujet, du contresujet, ou autre. Les plus scrupuleux noteront également les procédés

Transposition, 1 | 2011 
utilisés (canons divers, augmentation, mouvement contraire), les imitations au cours des divertissements et à chaque apparition du sujet, le rapport du ton employé avec la tonalité principale (ton relatif, sous-dominante, etc.). Les strettes employées dans le stretto sont dûment numérotées pour en suivre la progression. D'un simple coup d'œil, toute la structure de la composition apparaît donc clairement, permettant une prise de connaissance rapide et efficace.

\section{Les éléments thématiques}

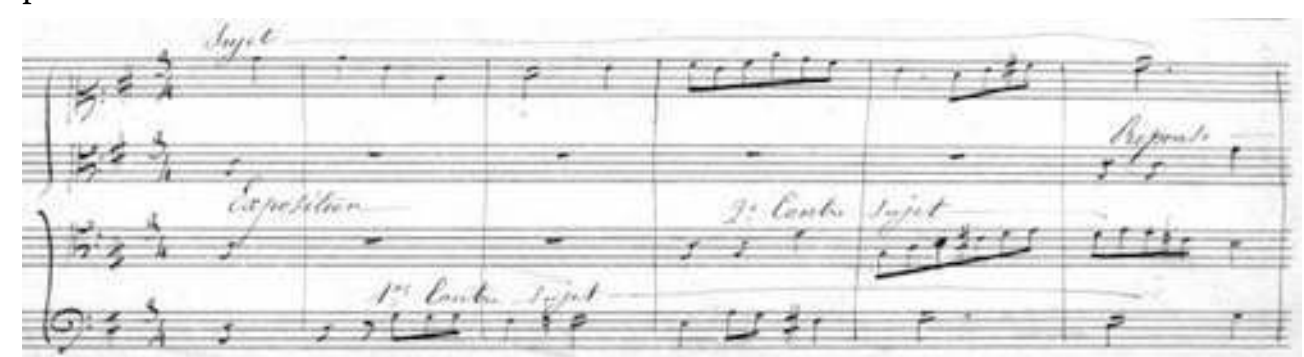


Le passage mélodique du sixième au septième degré du ton de ré majeur dans le second contresujet provoque inévitablement en si mineur la seconde augmentée. Cet intervalle ne peut pas être corrigé par l'emploi du mode mélodique au vu de la polyphonie :

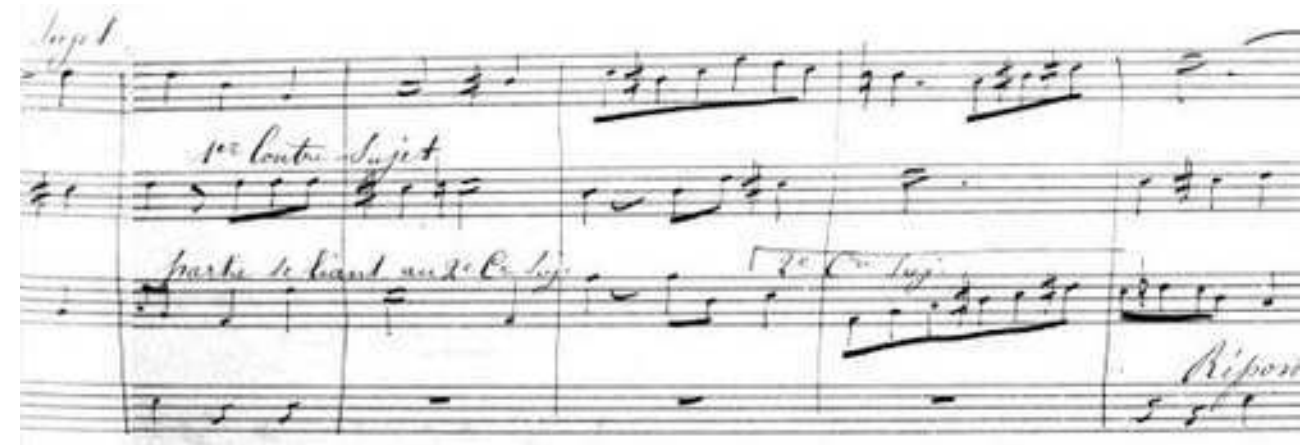

L'usage de la seconde augmentée n'est pas justifiable au vu des règles courantes du contrepoint. Manifestement, si Deslandres a pris la peine de contrôler le résultat du deuxième contresujet au relatif au moment de son élaboration, il a jugé ce défaut sans importance.

Ceci n'est pas un cas isolé à l'époque. Deux ans plus tard, Henri Fissot se heurte au même problème et modifie son contresujet à la fois rythmiquement et mélodiquement pour tenter d'adoucir le passage douteux (à gauche le contresujet original, à droite sa version modifiée) :
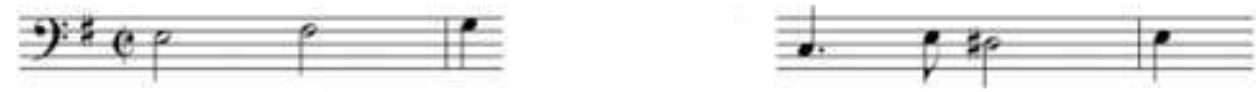

Parfaitement conscient qu'un tel changement n'a rien de régulier, le candidat s'en excuse par précaution en ajoutant sur sa copie : «j'ai cru pouvoir me permettre de faire quelques modifications dans le contresujet ».

Malgré des contresujets aussi évidemment défectueux, ces deux devoirs ont obtenu le premier prix, aussi faut-il conclure à une tolérance des jurys sur ce point précis.

\section{La réponse}

L'usage lors des concours de contrepoint et fugue est d'utiliser la réponse tonale, avec la mutation de tête le cas échéant. Il n'a pas été établi que les réponses réelle ou plagale soient interdites, mais il s'avère qu'elles n'ont pas été utilisées par les concurrents dans les cas ou elles étaient possibles. Nous en prendrons pour preuve les devoirs de l'année 1868. Le sujet donné cette année-là par Auber est le suivant :

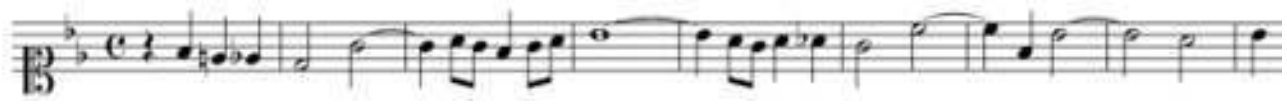

47 Le sujet commençant par la dominante $f a$ avant de se porter à la tonique si bémol mesure 4 , la réponse tonale commencera donc par la tonique pour se porter vers la dominante. C'est là qu'intervient un usage fort curieux : la mutation la plus pratique dans ce cas de figure consiste à laisser ce si bémol en place, jusqu'à rattraper sur la deuxième mesure l'intervalle de quinte par rapport au sujet et poursuivre ensuite à la quinte :

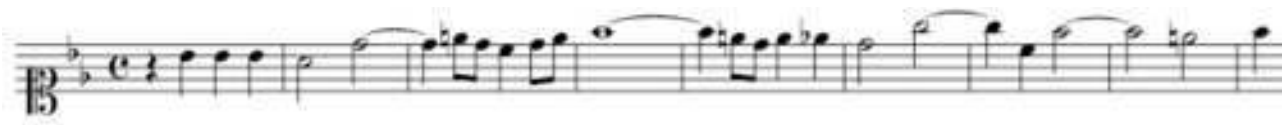


Les sept concurrents de l'année 1868 ont tous utilisé cette mutation. On s'aperçoit pourtant immédiatement de ce qu'elle a de vicieux musicalement, le caractère du motif de tête étant totalement dénaturé par l'élimination du mouvement chromatique. L'habitude prise de toujours écrire une fugue tonale, y compris lorsque le sujet donné ne s'y prête que médiocrement au point de vue musical, témoigne de la force du système reproductif de l'institution. Il faut attendre 1904 pour que Gedalge conseille dans son traité de préférer la réponse réelle à la réponse tonale dans ce cas de figure.

\section{Ordre de l'exposition}

Nous allons nous appuyer pour cette analyse sur les expositions des 58 fugues ayant obtenu le premier prix. Toutes les expositions respectent strictement l'alternance des tessitures : au sujet dans une voix aiguë (soprano ou ténor) correspond la réponse dans une voix grave (alto ou basse) et inversement.

Les expositions régulières, faisant entendre les entrées du sujet et de la réponse dans une partie qui, au moment de son entrée, se trouve être l'une des parties extrêmes de la polyphonie $^{19}$, ont été les plus employées. 46 lauréats ont choisi l'une de ces dispositions préférables et 12 seulement ont opté pour une disposition exceptionnelle. S'il n'est donc pas défendu d'user d'un ordre inhabituel, la tendance porte néanmoins vers le respect des usages établis comme étant les meilleurs.

51 Les trois quarts des sujets donnés aux concours sont écrits pour les voix aiguës. Le point le plus intéressant de ces expositions concerne le choix opéré dans ce cas entre le soprano et le ténor pour la première entrée. De 1858 à 1870, le ténor est choisi neuf fois, contre deux fois seulement pour le soprano. De 1895 à 1905, c'est le soprano qui est préféré dans sept copies, contre une seule pour l'entrée au ténor. Faire entrer le ténor en premier est donc plutôt une manière limitée au début de la période, car la tendance s'inverse en faveur du soprano dès la décennie 1870 .

52 Une telle disparité est révélatrice d'un changement de la conception du discours musical qui se fait au détriment de la richesse contrapuntique. En effet l'ordre d'entrée ténor, alto, soprano, basse implique le dispositif suivant :

S $\mathrm{S}$

A

R CS (partie libre)

T S CS (partie libre)

B

$\mathrm{R}$

Les éléments thématiques sont bien répartis dans les voix extrêmes, mettant en évidence le contrepoint renversable et l'alternance des voix. On entend successivement la réponse, le sujet et le contresujet comme partie supérieure de la polyphonie, mettant tous les éléments en valeur.

Le choix du soprano implique en revanche une prédominance de la partie mélodique principale qui chante en continu durant toute l'exposition et couvre les autres entrées. Le sujet et le contresujet y sont bien à découvert dès le début, mais plutôt dans un esprit d'affirmation que de dialogue. Le contresujet sera toujours au-dessus du sujet, au 
détriment du renversable. Précisons que ce choix n'implique aucune facilité technique : il faudra composer une partie libre mélodiquement intéressante sur les deux dernières entrées alors que la présence de deux parties obligées en dessous restreint les possibilités.

Nous assistons donc au même type d'évolution qu'en ce qui concernait la nature du travail contrapuntique: au désintérêt de la pratique du double contresujet vient s'adjoindre cette tendance à négliger le dialogue des voix et l'intérêt de toute la polyphonie, qui devrait pourtant être l'un des objectifs du travail de la fugue. Il semble donc que l'enseignement de cette discipline, compte tenu de l'éloignement croissant entre l'écriture d'école et la pratique de la composition dans un style contemporain, mette progressivement l'accent plutôt sur la qualité de l'enchaînement des idées et de leur gestion dans le temps musical que sur une maitrise de la pensée contrapuntique qui relève des esthétiques du passé.

\section{Pureté de l'écriture et respect du style rigoureux}

L'écriture de la fugue dans le style rigoureux implique le respect des règles du contrepoint sévère. Il semblerait cependant qu'une certaine indulgence ait été de mise vis-à-vis des négligences qui ont pu se glisser dans les devoirs. La nécessité d'éviter les quintes et octaves successives est une des règles de base d'une telle écriture. Il arrive pourtant que des fautes de cette nature se retrouvent encore dans les devoirs de prix. Pas moins d'une dizaine de ces fautes ont été ainsi signalées au crayon dans la fugue de Xavier Leroux en 1884, vraisemblablement par l'un des membres du jury. L'abondance de ces négligences n'a pas empêché Leroux d'obtenir le premier prix, ce qui signifie que la propreté de la réalisation n'est pas un critère fondamental du jugement porté sur les travaux. L'attention se focalise davantage sur le travail à grande échelle de la forme fuguée que sur les détails techniques, auxquels n'est pas accordée l'importance qu'ils paraissent avoir à la lecture des traités.

En revanche, le respect des règles inhérentes au style vocal de l'écriture semble faire partie des attentes du jury. Nous en prendrons pour exemple le contresujet réalisé par Debussy lors du concours de 1882, où il n'a obtenu qu'un deuxième accessit. Voici la première entrée du sujet, directement accompagné de son contresujet ${ }^{20}$ :

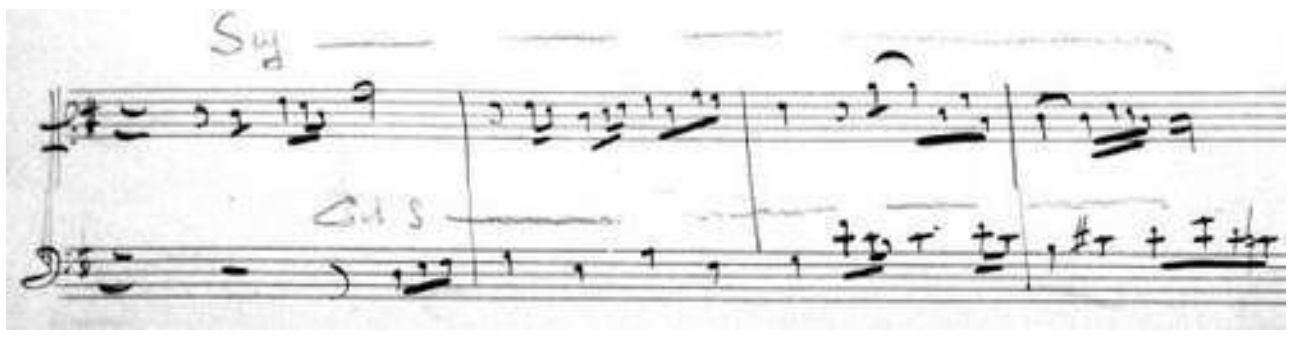

Le contresujet rentre donc par rapport au sujet en rapport d'octave (ou son redoublement). Le renversement des parties le fera rentrer sur un unisson, c'est-à-dire dans les deux cas une consonance parfaite. Or la note du sujet sur laquelle le contresujet débute sera mutée dans la réponse. Selon les règles, le contresujet aurait donc dû subir lui aussi une mutation correspondante, ce que Debussy choisit de ne pas faire en présentant ainsi l'entrée sur la réponse : 


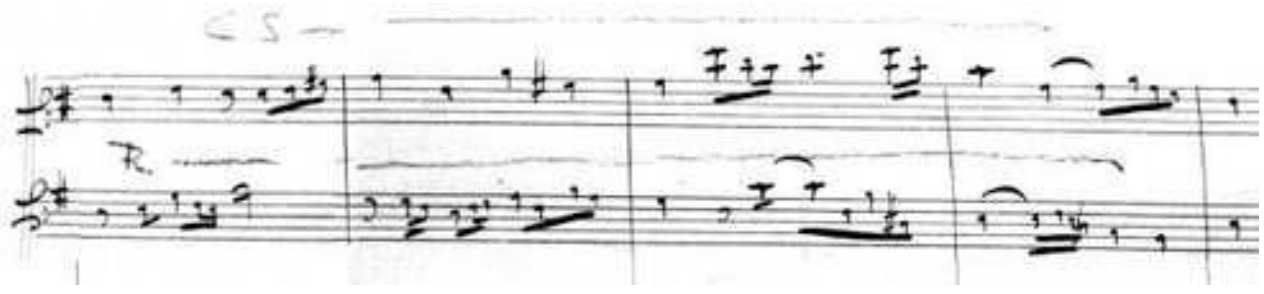
s'aperçoit tout de même que la candidate use de l'appoggiature assez librement. En revanche, après un silence simultané des quatre voix, le discours reprend sur la deuxième mesure par un accord de neuvième de dominante, la septième et la neuvième étant entendues sans préparation. La neuvième se résout sur l'octave sous forme de double appogiature, laquelle fait entendre avec le ténor une fausse relation chromatique. Le sol est une note étrangère à l'harmonie de dominante dont la fondamentale est si bémol. Enfin le si bécarre de la basse nous ramène vers do mineur mais reste en suspens à la fin de la mesure tandis que les autres voix se résolvent, créant une couleur d'accord augmenté. Cette écriture dans le style contemporain est totalement étrangère à tous les principes du contrepoint d'école qui est censé être mis en œuvre dans les fugues pour le concours. La simple analyse permet de repérer cette incompatibilité. L'élève ne se serait pas permis d'aussi grandes licences - qu'elle n'a pas pu laisser passer par négligence - si elle n'avait pas eu la certitude que cela ne lui serait pas reproché. De fait, le jury a mis son devoir en tête du palmarès. Cela s'explique par le contexte formel. La liberté d'écriture au moment 
de la pédale est plus grande que dans le reste de la composition. La manière d'enchaîner cette pédale au stretto n'est pas précisée dans les traités. La majorité des candidats se contentent d'une cadence avec repos général des voix. L'ajout ou non d'une formule de transition relève d'une initiative personnelle, il ne s'agit pas d'une attente liée à l'exercice de la fugue d'école. Les attentes fondamentales étant satisfaites, c'est donc aux marges de la forme, dans les enchaînements libres entre les parties conventionnelles que peuvent se glisser les licences. Debussy s'est autorisé une petite liberté sur un élément thématique fondamental en un moment crucial de la construction du discours fugué, celui de la mutation, et cela l'a desservi. Prestat a fait preuve d'une audace d'écriture bien plus grande, mais cette licence, placée en un endroit judicieux, ne lui a pas porté préjudice. On trouve ainsi souvent dans les devoirs, à partir des années 1880, de brefs passages disparates avec le reste de l'écriture, mais qui prennent place à des endroits stratégiques où les exigences techniques sont relâchées.

\section{Conclusion}

Au sein des disciplines contribuant à la formation d'un compositeur au Conservatoire, l'étude du contrepoint et de la fugue constitue l'un des versants les plus académiques. Au regard de l'histoire de l'institution, cet enseignement possédait déjà, à l'orée de la période qui nous intéresse, près d'un demi-siècle de tradition. Le processus à l'œuvre au moment de notre étude est celui de la cristallisation des éléments pédagogiques, capitalisant sur cette expérience acquise. Le système se replie alors sur lui-même, produisant en permanence la matière dont il a besoin pour entretenir son fonctionnement. La focalisation de l'intérêt sur les devoirs primés, qui culmine avec l'édition des meilleurs d'entre eux dans les traités au détriment des modèles du répertoire, est le signe de ce repli autarcique.

L'émergence de l'académisme dans l'enseignement de la fugue ne s'inscrit pourtant pas à l'origine dans une démarche réactionnaire. Le propos est de vivifier la création en s'abreuvant aux meilleures sources, et l'objet esthétique produit est un compromis nouveau, synthétique, hors de toute tradition historique et stylistique. Les œuvres du passé sont considérées à travers le prisme d'un regard contemporain. La rupture d'avec les modèles anciens, due à la distorsion de trois siècles d'évolution du langage et de la technique rassemblés en un même geste créateur, incite l'institution à se doter de ses propres modèles, conformes à ses attentes parce que conçus en fonction d'elles.

La personnalité musicale des étudiants qui suivent ce cursus est en revanche une variable au sein de la permanence de cet enseignement. Nourris dans un univers musical qui évolue au fil du temps, on ressent dans leur travail des tentatives de conciliation plus ou moins heureuses entre le monde contemporain et l'académisme de mise dans le cadre des concours. Tout en suivant la règle à la lettre, les meilleurs d'entre eux tenteront d'en contourner l'esprit en dégageant la partie mélodique du reste de la polyphonie, en étendant leur contrôle sur les tempi et les nuances, dimensions factices d'une œuvre abstraite et intellectualisée. L'expression personnelle se fait jour dès lors que la prescription est moins forte.

65 L'histoire de l'enseignement de la fugue dans la seconde moitié du XIX ${ }^{e}$ siècle doit donc aussi être celle des étudiants qui ont suivi cette formation, de laquelle sont sortis les musiciens les plus importants de leur temps. La permanence qui constitue le caractère essentiel de la pédagogie et de l'institution ne doit pas masquer les personnalités diverses 
qui ont été amenées à composer avec cette donnée. Cherchant tantôt à s'approprier une telle démarche, tantôt à la faire évoluer, ils ont toujours eu conscience du fait qu'un solide enseignement technique, quelles que puissent être les divergences sur son contenu, était la base essentielle d'une activité créatrice de haut niveau.

\section{BIBLIOGRAPHIE}

\section{Bibliographie}

\section{Sources}

ADAM, Adolphe, Souvenirs d'un musicien, Paris, Levy Frères, 1857.

CONSERVATOIRE NATIONAL DE MUSIQUE ET D'ART DRAMATIQUE DE PARIS, Distribution des Prix, Palmarès, Paris, 1834 - ...

DELDEVEZ, Marie-Edme-Ernest, Souvenirs d'études : harmonie, contrepoint, fugue, composition, Paris, Richault, 1877.

DUNAN, Elisabeth, Inventaire de la série AJ37, tome premier (1 à 375), Paris, Archives nationales, 1971.

FAURÉ, Gabriel, Correspondance, textes réunis, présentés et annotés par Jean-Michel Nectoux, Paris, Flammarion, 1980.

HALÉVY, Fromental, Lettres réunies et annotées par Marthe Galland, Heilbronn, Lucie Galland, 1999. HONDRÉ, Emmanuel, « Liste des professeurs des origines à nos jours » in HONDRÉ, Emmanuel (ed.), Le conservatoire de musique de Paris, Paris, Association du bureau des étudiants du CNSMDP, 1995.

LASSABATHIE, Théodore, Histoire du Conservatoire impérial de musique et de déclamation; suivie de documents recueillis et mis en ordre, Paris, Levy Frères, 1860.

MASSENET, Jules, Mes souvenirs, Paris, P. Lafitte, 1912.

PIERRE, Constant, Le Conservatoire national de musique et de déclamation. Documents historiques et administratifs, Paris, Imprimerie nationale, 1900.

PIERRE, Constant, Sujets de fugue et thèmes d'improvisation donnés aux concours d'essai pour le grand prix de Rome, aux concours de fugue, et aux examens d'orgue, années 1804-1900:288 sujets, Paris, Heugel, 1900.

\section{Traités}

MARPURG, Friedrich-Wilhelm, Traité de la fugue et du contrepoint, Paris, Imbault, 1801.

LANGLÉ, Honoré, Traité de la fugue, Paris, l’Auteur, 1805.

REICHA, Anton, Traité de haute composition musicale, Paris, Zetter, 1824. 
FÉTIS, François-Joseph, Traité du contrepoint et de la fugue, Paris, 1825.

CHERUBINI, Luigi, Cours de contrepoint et de fugue, Paris, Schlesinger, 1835.

ELWART, Antoine, Le contrepoint et la fugue appliqués à la composition idéale, Paris, Lemoine, 1840.

ZIMMERMAN, Pierre-Joseph, Traité d'harmonie, du contrepoint et de la fugue : la composition appliquée à l'orchestre et au théâtre : ouvrage composé pour le Conservatoire de Paris, Paris, ca. 1843.

BAZIN, François-Emmanuel-Joseph, Cours de contrepoint théorique et pratique, Paris, Lemoine, 1881.

BISSON, Alexandre, Petit traité de composition musicale : composition, mélodie, harmonie, contrepoint, fugue, instrumentation, voix, exécution, Paris, Hennuyer, 1881.

DUBOIS, Théodore, Traité de contrepoint et de fugue, Paris, Heugel, 1901.

GEDALGE, André, Traité de la fugue, Paris, Enoch et Cie, 1901.

\section{Études}

BARTOLI, Jean-Pierre, L'harmonie classique et romantique, 1750-1900, éléments et évolution, Paris, Minerve, 2001.

FAYOLLE, Coralie, « Les traités de contrepoint en France aux $\mathrm{XIX}^{\mathrm{e}}$ et $\mathrm{XX}^{\mathrm{e}}$ siècles : permanence et renouvellement ", in BONGRAIN, Anne-Marie, POIRIER, Alain (ed.), Le Conservatoire de Paris, 1795-1995, deux cents ans de pédagogie, Paris, Buchet-Chastel, 1999.

JUTTEN, Odile, L'enseignement de l'improvisation à la classe d'orgue du Conservatoire de Paris : 1819-1986, d'après la thématique de concours et d'examen, thèse, Université Paris IV-Sorbonne, 1999.

MANN, Alfred, Theory and Practice, The Great Composers as Student and Teacher, New-York, Norton \& Cie, 1987.

NECTOUX, Michel, « Tous écoutent la parole du maître : Gabriel Fauré et ses élèves » in BONGRAIN, Anne-Marie, POIRIER, Alain (ed.), Le Conservatoire de Paris, 1795-1995, deux cents ans de pédagogie, Paris, Buchet-Chastel, 1999.

NICEPHOR, Sylvie, L'apprentissage de la composition musicale : regard sur la situation française durant la première moitié $d u \mathrm{XIX}^{e}$ siècle, thèse, Université Paris IV-Sorbonne, 2007.

PIERRE, Constant, B. Sarrette et les origines du Conservatoire national de musique et de déclamation, Paris, Delalain Frères, 1895.

PIERRE, Constant, « Le Conservatoire national de musique », in La Revue musicale, Paris, 1903.

RAMAUT, Alban, « Anton Reicha et le Conservatoire » in BONGRAIN, Anne-Marie, POIRIER, Alain (ed.), Le Conservatoire de Paris, 1795-1995, deux cents ans de pédagogie, Paris, Buchet-Chastel, 1999.

RONXIN, Nathalie, «Les classes d'écriture et de composition : cursus complémentaires ou parallèles ? ", in HONDRÉ, Emmanuel (ed.), Le conservatoire de musique de Paris, Paris, Association du bureau des étudiants du CNSMDP, 1995.

\section{NOTES}

1. HONDRÉ, Emmanuel (ed.), Le conservatoire de musique de Paris, Paris, Association du bureau des étudiants du CNSMDP, 1995. BONGRAIN, Anne-Marie et POIRIER, Alain (ed.), Le Conservatoire de Paris, 1795-1995, deux cents ans de pédagogie, Paris, Buchet-Chastel, 1999.

2. Le nombre de classes sera réduit à trois en 1869. 
3. Pour une liste complète des enseignants, nous renvoyons à : HONDRÉ, Emmanuel, « Liste des professeurs des origines à nos jours » in HONDRÉ, Emmanuel (ed.), op. cit.

4. PIERRE, Constant, Le Conservatoire national de musique et de déclamation. Documents historiques et administratifs, Paris, Imprimerie Nationale, 1900, p. 255.

5. PIERRE, Constant, op. cit., p. 260.

6. Liste des lauréats établie d'après : Conservatoire national de musique et d'art dramatique de Paris, Distribution des Prix, Palmarès, Paris, 1834 - ...

7. PIERRE, Constant, op. cit., p. 684-872.

8. Informations tirées des notes de jury prises par Théodore Dubois entre 1879 et 1891, Archives Nationales, Paris, cote AJ37-237-1.

9. Document conservé aux Archives Nationales, Paris, série AJ37, cote 296. Il est écrit: "Très intelligent, très doué, mais trop recherché, trop raffiné. Élève assidu".

10. Déroulement des jugements reconstitué d'après les procès-verbaux des séances du jury, Archives Nationales, série AJ37, cotes 250 à 253.

11. GEDALGE, André, Traité de la fugue, Paris, Enoch \& Cie, 1901.

12. Concernant l'études des traités voir également: FAYOLLE, Coralie, «Les traités de contrepoint en France aux XIX et $\mathrm{XX}^{\mathrm{e}}$ siècles : permanence et renouvellement », in BONGRAIN, Anne-Marie et POIRIER, Alain (ed.), op. cit.

13. PIERRE, Constant, op. cit., p. 229.

14. FÉTIS, François-Joseph, op. cit., p. 137.

15. REICHA, Anton, op. cit., n.p.

16. Tous ces devoirs sont conservés aux Archives Nationales, Paris, série AJ37, cotes 204-10 à 204-31.

17. Par ex. : PIERRE, Constant, Sujets de fugue et thèmes d'improvisation donnés aux concours d'essai pour le grand prix de Rome, aux concours de fugue, et aux examens d'orgue, années 1804-1900 : 288 sujets, Paris, Heugel, 1900.

18. Document conservé aux Archives Nationales, cote AJ37-204-10.

19. À savoir : soprano, alto, ténor, basse ou ténor, alto, soprano, basse pour un sujet en voix aiguë, et basse, ténor, alto, soprano ou alto, ténor, basse, soprano pour un sujet en voix grave.

20. Document conservé aux Archives Nationales, cote AJ37-204-16.

21. Document conservé aux Archives Nationales, cote AJ37-204-21.

\section{RÉSUMÉS}

Le propos est de restituer le projet pédagogique de ces disciplines et les conceptions du contrepoint et de la fugue que cela sous-tend en interrogeant les documents d'époque. Après avoir décrit l'organisation institutionnelle de l'enseignement, le contenu des ouvrages pédagogiques publiés par les professeurs du Conservatoire sera questionné afin d'appréhender la démarche adoptée pour la structuration de l'enseignement et la sélection du corpus de référence. L'analyse des fugues réalisées par les élèves eux-mêmes à l'occasion du concours annuel des prix permettra de saisir concrètement l'aboutissement de cette pédagogie. On pourra par la confrontation de ces sources tenter de comprendre le concept d'écriture contrapuntique tel qu'on l'envisage en France à cette époque. 
This article attempts to describe the pedagogic project about counterpoint and fugue, and considers it in its context. The used documents are mostly historical sources, especially archives from the Conservatoire. The reconstitution of the considered teachings will be done in three points. First of all, the institutional organisation will be studied to situate the learning of these subjects as a part of a global formation for young musicians, specially destined to future composers. In a second time, our attention will be concentrated on the treatises published by the teachers of the Conservatoire themselves, often used in the classes, as a witnesses of their teaching and their pedagogy. Finally, the analysis of fugues written by students for their exam will inform us about the concrete result of the studies in counterpoint and fugue during these years.

\section{INDEX}

Keywords : contest, counterpoint, education, pedagogy, award, treatise, 19th century, composition, Conservatoire de Paris, fugue, Paris

Mots-clés : concours, contrepoint, enseignement, pédagogie, prix, traité, XIXe siècle, composition, Conservatoire de Paris, fugue, Paris

\section{AUTEUR}

\section{ANTHONY BERGERAULT}

Après une licence de musicologie, il poursuit des études d'écriture musicale au CNSMD de Paris, au cours desquelles il obtient six prix. Complétant sa formation dans les classes d'analyse,

d'histoire de la musique et d'esthétique, il choisit d'orienter ses recherches vers la pédagogie de l'écriture. Il intègre en 2010 le CNSMD de Lyon pour la formation au CA dans cette discipline. 